\title{
SOLUTION OF AN INTEGRAL EQUATION WITH A LOGARITHMIC KERNEL
}

\author{
C. SAMPATH and D.J. JAIN \\ Department of Mathematics \\ Faculty of Mathematical Sciences \\ University of Delhi \\ Delhi - 110007, India \\ (Received July 14, 1989 and in revised form January 7, 1990)
}

\begin{abstract}
A simple independent integral equation technique is presented to solve an integral equation with a logarithmic kernel which governs solutions of many twodimensional Dirichlet boundary value problems involving two coplanar and parallel infinite strips. The technique is further fllustrated by an example.
\end{abstract}

KEY WORDS AND PHRASES. Coplanar and parallel infinite strips, logarithmic kernel, elliptic integrals of the first, the second and third kinds.

1980 AMS SUBJECT CLASSIFICATION CODE. 45.

1. INTRODUCTION.

Solutions of many two-dimensional Dirichlet boundary value problems involving two equal coplanar and parallel strips: $0<b<|x|<a, y=0,-\infty<z<\infty$, are governed by a Fredholm integral equation of the first kind with logarithmic kernel $\left\{2 p+\log \left|4\left(x^{2}-x_{1}^{2}\right)\right|\right\}, b<x, x_{1}<a$, in which the known function as well as the unknown function are even degree functions and $p$ is a known constant.

In order to solve this integral equation, both sides are first differentiated with respect to $x^{2}$ to reduce it into a singular integral equation with kernel $\left(x^{2}-x_{1}^{2}\right)^{-1}$ which can be easily inverted by the known technique (Lowengrub and Srivastava [1] and Jain and Kanwal [2]. The solution of this singular integral equation contains an unknown constant which is evaluated by substituting this solution in the original integral equation with logarithmic kernel. Unfortunately, the process of evaluating this unknown constant is very tedious.

We present here a simple independent technique to solve a Fredholdm integral equation of the first kind with logarithmic kernel $\left\{2 p+\log \left|4\left(x^{2}-x_{1}^{2}\right)\right|\right\}, b<x$, $x_{1}<a$, in which the known function as well as the unknown function are even degree functions and $p$ is a known constant. By simple substitutions, this equation is first 
transformed into a Fredholm integral equation of the first kind with logarithmic kernel $\left\{2 q+\log \left|2\left(\cos \theta-\cos \theta_{1}\right)\right|\right\}, 0<\theta, \theta_{1}<\pi$ where $q$ is a known constant. Finally using the Fourier series expansions of the known function of this integral equation and its kernel, the constant coefficients in the Fourler series expansion of its unknown function are readily obtained by appealing to the orthogonal property of cosines of the multiples of angle $\theta$ over the interval $[0, \pi]$. Thus we have obtained the Fourier series expansion of the unknown function of this integral equation which readily yields the required solution of the given Fredholm integral equation by appealing to the substitutions already used. We also explain here that this series solution of the given integral equation can be reduced to the closed form obtained by the known methods $[1,2]$. Finally we 1llustrate the application of our technique to solve the Fredholm integral equation of the first kind with logarithmic kernel $\left.\{2 p+\log \mid 4)\left(x^{2}-x_{1}^{2}\right) \mid\right\}, b<x, x_{1}<a, p$ being a known constant when the known even degree function is $|x|$.

\section{INTEGRAL EQUATION TECHNIQUE.}

Solutions of many two-dimension Dirichlet boundary value problems involving two equal coplanar and parallel strips: $0<b<|x|<a, y=0,-\infty<z<\infty$, is governed by an integral equation of the form

$$
\int_{-a}^{-b}+\int_{b}^{a} g\left(x_{1}^{2}\right)\left\{p+\log 2\left|x-x_{1}\right|\right\} d x_{1}=\frac{\pi}{2} f\left(x^{2}\right), b<|x|<a
$$

where $f\left(x^{2}\right)$ is a known even degree function, $p$ is a constant and $g\left(x_{1}^{2}\right)$ is an unknown even degree function.

When we substitute in the integral equation (2.1)

$$
g\left(x_{1}^{2}\right)=\left|x_{1}\right| \phi\left(x_{1}^{2}\right), \quad b<\left|x_{1}\right|<a,
$$

it takes a simpler form

$$
\int_{b}^{a} x_{1} \phi\left(x_{1}^{2}\right)\left\{2 p+\log \left|4\left(x^{2}-x_{1}^{2}\right)\right|\right\} d x_{1}=\frac{\pi}{2} f\left(x^{2}\right), \quad b<x<a .
$$

In order to solve this integral equation by the known method $[1,2]$, we first differentiate its both sides with respect to $x^{2}$ and get,

$$
\int_{b}^{a} \frac{x_{1} \phi\left(x_{1}^{2}\right) d x_{1}}{x^{2}-x_{1}^{2}}=\frac{\pi}{2} f^{\prime}\left(x^{2}\right), \quad b<x<a,
$$

where the singular integral is a Cauchy principal value. The solution of this integral equation 18 known to be $[1,2]$ 


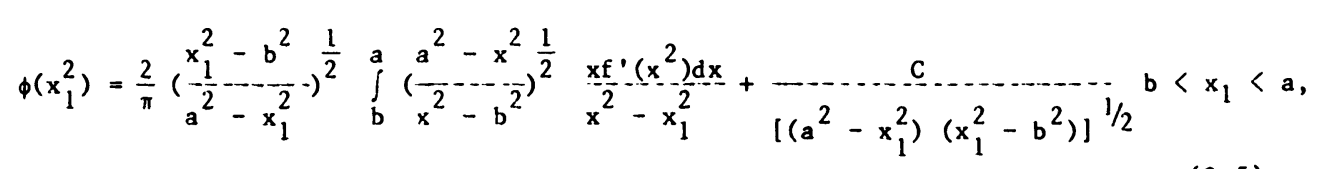

where the constant $C$ is to be evaluated by putting this value of $\phi\left(x_{1}^{2}\right)$ in (2.3). This process of evaluation of the constant $C$ is very tedious as clearly shown in the illustrated example given at the end.

We present here a simple independent method of solving the integral equation (2.3) without differentiating its both sides. First in (2.3) we make the substitutions

$$
\begin{aligned}
& x_{1}^{2}=A \cos \theta_{1}+B, \\
& x^{2}=A \cos \theta+B,
\end{aligned}
$$

where

$$
A=\frac{a^{2}-b^{2}}{2}, B=\frac{a^{2}+b^{2}}{2},
$$

$0<\theta, \theta_{1}<\pi$ when $b<x, x_{1}<a$, and get

$$
\int_{0}^{\pi} h\left(\theta_{1}\right)\left\{2 q+\log \left|2\left(\cos \theta-\cos \theta_{1}\right)\right|\right\} d \theta_{1}=\frac{\pi}{2} F(\theta), \quad 0<\theta<\pi
$$

where

$$
\begin{aligned}
& h\left(\theta_{1}\right)=\frac{A}{2} \sin \theta_{1} \phi\left(x_{1}^{2}\right), \\
& q=p+\log \left(a^{2}-b^{2}\right)^{1 / 2}, \\
& F(\theta)=f\left(x^{2}\right) .
\end{aligned}
$$

Now the Fourier series expansion of the known function $F(\theta)$

where

$$
\frac{\pi}{2} F(\theta)=\frac{1}{2} C_{0}+\sum_{n=1}^{\infty} C_{n} \cos n \theta, \quad 0<\theta<\pi,
$$

$$
\begin{aligned}
C_{n} & =\int_{0}^{\pi} F(\theta) \cos n \theta d \theta \\
& =\int_{0}^{\pi} f\left(x^{2}\right) \cos n \theta d \theta, \quad n=0,1,2, \ldots
\end{aligned}
$$

are known coefficients and the formula

$$
\log \left|2\left(\cos \theta-\cos \theta_{1}\right)\right|=-2 \sum_{n=1}^{\infty} \frac{\cos n \theta \cos n \theta_{1}}{n}, 0<\theta, \theta_{1}<\pi,
$$

lead the integral equation (2.8) to the form 


$$
\begin{aligned}
\int_{0}^{\pi}\left\{\frac{1}{2} d_{0}\right. & \left.+\sum_{1}^{\infty} d_{n} \cos n \theta_{1}\right\}\left\{2 q-2 \sum_{n=1}^{\infty} \frac{\cos n \theta \cos n \theta_{1}}{n} d \theta_{1}\right. \\
& =\frac{1}{2} c_{0}+\sum_{n=1}^{\infty} c_{n} \cos n \theta, \quad 0<\theta<\pi,
\end{aligned}
$$

where we have used the following Fourier series expansion of the unknown function $h\left(\theta_{1}\right)$ :

$$
h\left(\theta_{1}\right)=\frac{1}{2} d_{0}+\sum_{n=1}^{\infty} d_{n} \cos n \theta_{1}, \quad 0<\theta_{1}<\pi .
$$

The values of the constant coefficients $d_{n}, n=0,1,2, \ldots$ in this expansion are readily obtalned from equation (2.14) in terms of the known coefficients $C_{n}$, $n=$ $0,1,2, \ldots$ by appealing to the orthogonal property of cosines of the multiples of angle $\theta_{1}$ over the interval $0<\theta_{1}<\pi$. These values are

$$
d_{0}=\frac{C_{0}}{2 \pi q}, d_{n}=-\left(n C_{n}\right) / \pi, \quad n>1,
$$

where the values of the known coefficients $C_{n}$ are given in relations (2.12). Lastly, using the relations (2.9), (2.15) and (2.16) we obtain the required value of the unknown function $\phi\left(x_{1}^{2}\right)$ of the integral equation (2.3) in the form

$$
\phi\left(x_{1}^{2}\right)=\frac{2}{\pi\left[\left(a^{2}-x_{1}^{2}\right)\left(x_{1}^{2}-b^{2}\right)\right]}\left\{C_{0} /(4 q)-\sum_{n=1}^{\infty} n C_{n} \cos n \theta_{1}\right\},
$$

where the relation between $\cos \theta_{1}$ and $x_{1}^{2}$ is given in the substitutions (2.6) and we have used the following formulas readily derived from this relation:

$$
\cos \frac{\theta_{1}}{2}=\left(\frac{x_{1}^{2}-b^{2}}{a^{2}-b^{2}}\right)^{1 / 2}, \sin \frac{\theta_{1}}{2}=\left(\frac{a^{2}-x_{1}^{2}}{a^{2}-b^{2}}\right)^{1 / 2} \text {. }
$$

We now explain that the series solution (2.17) of the unknown function $\phi\left(x_{1}^{2}\right)$ can be put in the closed form (2.5) which 18 derived by the known technique [1,2] and contains an unknown constant $C$. When we integrate by parts, we obtain the integrals which define the values of the $C_{n}$ in relations $(2.12)$, as

$$
C_{n}=\frac{A}{n} \int_{0}^{\pi} \sin n \theta \cdot \sin \theta \cdot f^{\prime}\left(x^{2}\right) d \theta, \quad n>1,
$$

where we have used the relation between $x^{2}$ and $\cos \theta$ defined in the substitutions (2.6). Substitution of these values of the coefficients $C_{n}, n>1$ in the series solution (2.17), gives

$$
\phi\left(x_{1}^{2}\right)=\frac{2}{\pi\left[\left(a^{2}-x_{1}^{2}\right)\left(x_{1}^{2}-b^{2}\right)\right]^{1 / 2}}\left\{\frac{1}{4 q}-\int_{0}^{\pi} f\left(x^{2}\right) d \theta+\frac{A}{2} \int_{0}^{\pi}(1-\cos \theta) f^{\prime}\left(x^{2}\right) d \theta\right.
$$




$$
+\frac{A}{2}\left(1+\cos \theta_{1}\right) \int_{0}^{\pi} \tan \frac{\theta}{2} \sin \theta \cdot \frac{f^{\prime}\left(x^{2}\right) d \theta}{(\cos \theta}-\frac{\left.\cos \theta_{1}\right)}{d}, b<x_{1}<a,
$$

where we have used the relation

$$
\begin{aligned}
\sum_{n=1}^{\infty} \sin n \cos n \theta_{1} & =-\frac{1}{2}\left(-\frac{\sin \theta}{\cos \theta-\cos \theta}\right) \\
& =-\frac{1}{2}\left(\frac{\sin \theta}{1+\cos \theta}\right)\left(1+\frac{1+\cos \theta_{1}}{\cos \theta-\cos \theta_{1}}\right), \quad 0<\theta_{1}, \theta_{1}<\pi,
\end{aligned}
$$

readily derived from the formula (2.13) by differentiating both sides with respect to $\theta$. Finally, when we use the substitutions (2.6), relations (2.7) and (2.18) in the expression (2.20), we obtain

$$
\begin{aligned}
& \phi\left(x_{1}^{2}\right)=\frac{2}{\pi\left[\left(a^{2}-x_{1}^{2}\right)\left(x_{1}^{2}-b^{2}\right)\right]} \frac{1 / 2}{2 q} \int_{b}^{a} \frac{x f\left(x^{2}\right) d x}{\left[\left(a^{2}-x^{2}\right)\left(x^{2}-b^{2}\right)\right]^{1 / 2}} \\
& +\int_{b}^{a}\left(\frac{a^{2}-x^{2}}{x^{2}-b^{2}}\right)^{1 / 2}\left(x f^{\prime}\left(x^{2}\right)\right) d x \\
& \left.+\left(x_{1}^{2}-b^{2}\right) \int_{b}^{a} \frac{a^{2}-x^{2}}{x^{2}-b^{2}}\right) \frac{1 / 2 \frac{\left(x f^{\prime}\left(x^{2}\right)\right) d x}{2}-\frac{x_{1}^{2}}{x^{2}-x_{1}}, \quad b<x_{1}<a,}{}
\end{aligned}
$$

which agrees with the known result (2.5), and the value of the unknown constant $C$ in the known result (2.5) is thus also evaluated by our technique and its value is

$$
C=\frac{2}{\pi}\left\{\frac{l}{2 q} \int_{b}^{a} \frac{x f\left(x^{2}\right) d x}{\left[\left(a^{2}-x^{2}\right)\left(x^{2}-b^{2}\right)\right]}-\frac{1 / 2}{b}+\int_{b}^{a}\left(\frac{a^{2}-x^{2}}{x^{2}-b^{2}}\right)^{1 / 2} x f \cdot\left(x^{2}\right) d x\right\}
$$

This appears to be a new result.

\section{AN ILLUSTRATIVE EXAMPLE.}

To illustrate our technique, we solve the integral equation (2.3) when

$$
f\left(x^{2}\right)=|x|=\sqrt{x^{2}}
$$

80 in this case

$$
f(x)=\sqrt{x}, f^{\prime}(x)=\frac{1}{2 \sqrt{x}}, f^{\prime}\left(x^{2}\right)=\frac{1}{2 \sqrt{x^{2}}}
$$

Solution of this equation by the known technique [2] is obtained by using the formula (2.5) and in this case, we have

$$
\begin{aligned}
\phi\left(x_{1}^{2}\right)=\frac{1}{2 \pi a}\left(\frac{x_{1}^{2}-b^{2}}{a^{2}-x_{1}^{2}}\right) & 1 / 2 \int_{0}^{\pi} \frac{\sin ^{2} \frac{\theta}{2} d \theta}{\left(\sin ^{2} \frac{1}{2}-\sin ^{2} \frac{\theta}{2}\right)\left[1-k^{2} \sin ^{2} \frac{\theta}{2}\right]^{1 / 2}} \\
& +\frac{C}{\left[\left(a^{2}-x_{1}^{2}\right)\left(x_{1}^{2}-b^{2}\right)\right]} \frac{1 / 2}{1 / 2}
\end{aligned}
$$




$$
\begin{aligned}
& =\frac{1}{\pi a}-\left(\frac{x_{1}^{2}-b^{2}}{a^{2}-x_{1}^{2}}\right) 1 / 2\left[\pi\left(\frac{\pi}{2}, \frac{a^{2}-b^{2}}{a^{2}-x_{1}^{2}},\left(1-c^{2}\right)^{1 / 2}\right]-F\left(\frac{\pi}{2},\left(1-c^{2}\right)^{1 / 2}\right)\right] \\
& +\frac{c}{\left[\left(a^{2}-x_{1}^{2}\right)\left(x_{1}^{2}-b^{2}\right)\right]} 1 / 2, b<x_{1}<a,
\end{aligned}
$$

where we have used the substitutions $(2.6)$, the relations $(2.18)$, and is the elliptic integral of the third kind $\pi$ :

$$
\Pi\left(\theta, n^{2}, K\right)=\int_{0}^{\theta} \frac{d \alpha}{\left(1-n^{2} \sin ^{2} \alpha\right)\left(1-k^{2} \sin ^{2} \alpha\right)^{1 / 2}},
$$

$k^{2}=1-c^{2}, c=b / a$, and $F\left(\frac{\pi}{2}, k\right)$ is the elliptic integral of the first kind.

In this case the solution $\phi\left(x_{1}^{2}\right)$ of the integral equation (2.3) given by the equation (3.2) still contains the unknown constant $C$ which is finally evaluated by substituting this value of $\phi\left(x_{1}^{2}\right)$ in the integral equation (2.3) and evaluating some complicated definite integrals.

The required value of $C$, in this case, is given by [2]

$$
C=\frac{a}{\pi}\left[\left(\frac{1}{q}-1\right) E\left(\frac{\pi}{2}, K\right)+F\left(\frac{\pi}{2}, K\right)\right],
$$

where $E\left(\frac{\pi}{2}, K\right)$ is the elliptic integral of the second kind.

By substituting the above value of the constant $C$ in the equation (3.2), we obtain the required solution of the integral equation (2.3), in this case, by the known technique $[1,2]$. We readily obtain the solution of the integral equation (2.3) by our technique, in this case, by putting the values of $f\left(x^{2}\right), f^{\prime}\left(x^{2}\right)$ from the relations (3.1) in the formula (2.22) and this is given by

$$
\begin{aligned}
& \phi\left(x_{1}^{2}\right)=\frac{2 a}{\left.\pi\left[a^{2}-x_{1}^{2}\right)\left(x_{1}^{2}-b^{2}\right)\right]} 1 / 2\left\{\frac{1}{2 q} E\left(\frac{\pi}{2}, K\right)+\frac{1}{2}\left[F\left(\frac{\pi}{2}, K\right)-E\left(\frac{\pi}{2}, K\right)\right]\right. \\
& \left.+\frac{1}{2 a^{2}}\left(x_{1}^{2}-b^{2}\right)\left[\pi\left(\frac{\pi}{2}, \frac{a^{2}-b^{2}}{a^{2}-x_{1}^{2}}, K\right)-F\left(\frac{\pi}{2}, K\right)\right]\right\}, \quad b<x_{1}<a .
\end{aligned}
$$

This agrees with the solution given by the equations (3.2) and (3.4) which was derived by the known technique $[1,2]$.

\section{REFERENCES}

1. LOWENGRUB, M. and SRIVASTAVA, K.N., Research Note on Two Coplanar Griffith Cracks in an Infinite Elastic Medium, Int. J. Engng. Sc1. 6 (1968), 359-262.

2. JAIN, D.L. and KANWAL, R.P., Acoustic Diffraction of a Plane Wave by Two Coplanar Parallel Perfectly Soft or Rigid Strips Can. J. Phys. 50 (1972), 929-939. 


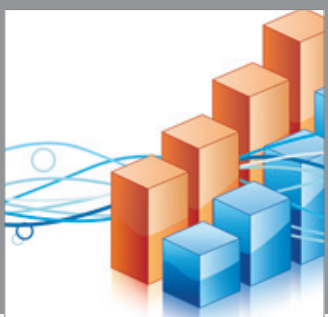

Advances in

Operations Research

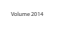

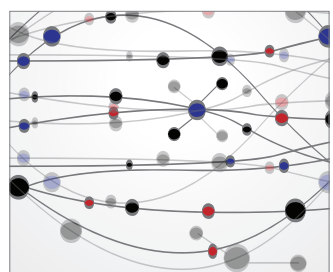

\section{The Scientific} World Journal
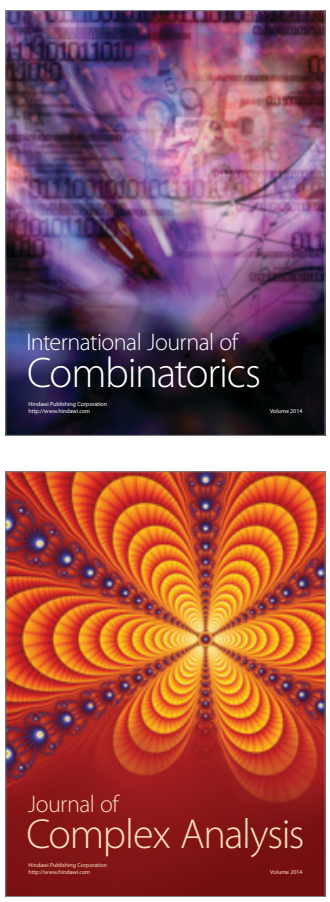

International Journal of

Mathematics and

Mathematical

Sciences
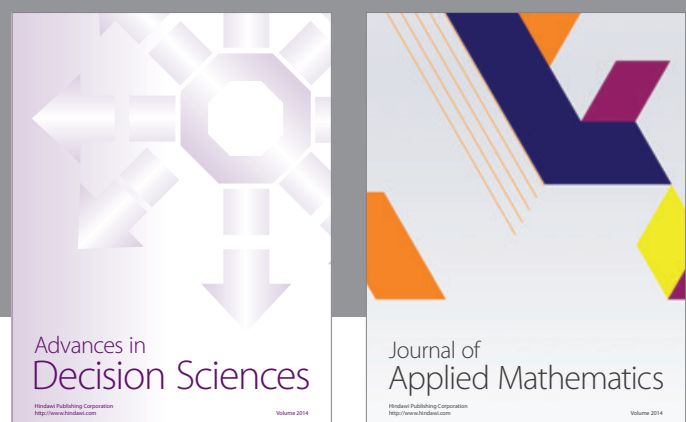

Journal of

Applied Mathematics
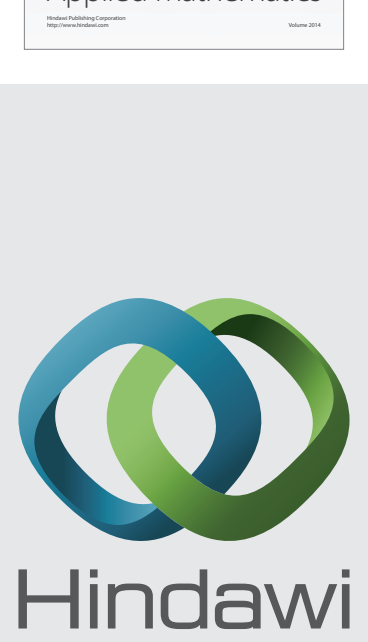

Submit your manuscripts at http://www.hindawi.com
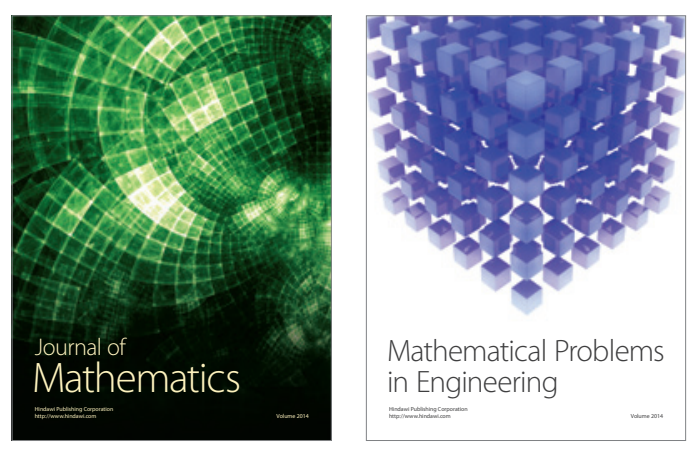

Mathematical Problems in Engineering
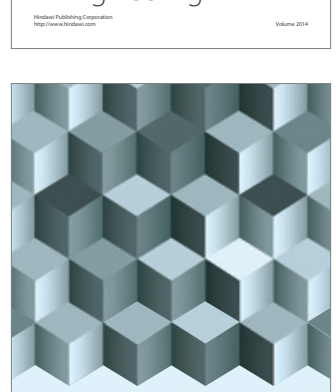

Journal of

Function Spaces
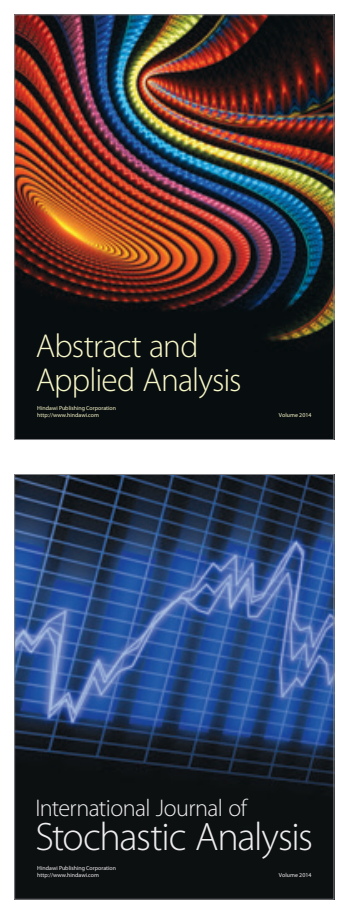

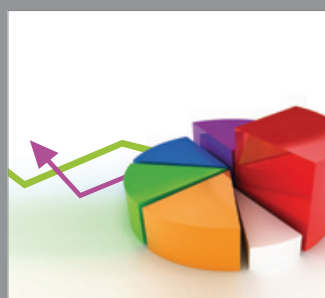

ournal of

Probability and Statistics

Promensencen
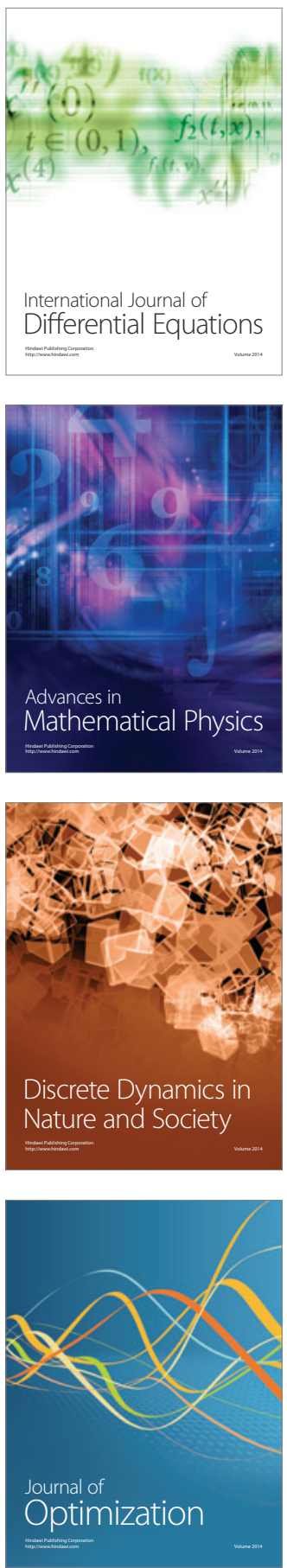